\title{
¿SON LOS E-SPORTS UN DEPORTE? EL TÉRMINO ‘DEPORTE’ EN JAQUE
}

\author{
O E-SPORTS É UM ESPORTE? O TERMO “ESPORTE” EM CHEQUE
}

ARE E-SPORTS A SPORT? THE TERM 'SPORT' IN CHECKMATE

\author{
Antonio Bascón-Seda*, Gonzalo Ramírez-Macías *
}

Palabras clave: e-sports.

Terminología. Juegos de video. Sociología.

Palavras chave: e-sports. Terminologia. Jogos de vídeo. Sociologia.

\section{Keywords:} e-sports. Terminology. Video games. Sociology.
Resumen: Este ensayo busca analizar de forma crítica el término deporte a partir de su definición y sus características. De esta forma, se trata el tan controvertido tema de si los deportes electrónicos (e-sports) pudieran formar parte del concepto deporte. A partir de esta idea se pretende provocar la reflexión y el debate con respecto a la consistencia, claridad y precisión del término deporte. Como conclusiones, se defiende que los e-sports podrían considerarse deportes según la definición actual del término, si bien la conclusión más relevante es que el concepto deporte necesita de una nueva definición donde se clarifiquen sus características básicas en relación al contexto actual.

Resumo: Este ensaio procura analisar criticamente o termo esporte a partir de sua definição e características. Desta forma, trata da questão altamente controversa de saber se o esporte eletrônico (e-sports) poderia fazer parte do conceito de esporte. Com base nesta ideia, pretende-se provocar reflexão e debate sobre a consistência, clareza e precisão do termo esporte. Como conclusões, argumentase que o esporte eletrônico poderia ser considerado esporte de acordo com a definição atual do termo, embora a conclusão mais relevante seja que o conceito de esporte necessita de uma nova definição nas qual as suas características básicas sejam clarificadas em relação ao contexto atual.

Abstract: This essay seeks to critically analyze the term sport from its definition and characteristics. Therefore, it is the highly controversial issue of whether electronic sports (e-sports) could be part of the concept of sport. Based on this idea, the work intends to provoke reflection and debate on the consistency, clarity, and precision of the term sport. As conclusion, it is argued that e-sports could be considered sports according to the current definition of the term, although the most relevant conclusion is that the concept of sport needs a new definition where its basic characteristics are clarified in the current context.
*Universidad de Sevilla, Sevilha, Espanha. E-mail: antoniobascon@us.es; grm@us.es

Recebido em: 14-10-2019 Aprovado em: 08-06-2020 Publicado em: 27-06-2020 (c) (i) () Licence 


\section{INTRODUCCIÓN}

Internet y su expansión global, marcan una nueva época conocida como sociedad de la información, caracterizada por el uso cotidiano de las Tecnologías de la Información y la Comunicación (TIC) y su influencia sobre el resto de áreas y dimensiones sociales del ser humano (LAFRANCE, 2003). De esta forma, se han originado nuevas estructuras sociales que han modificado la concepción del videojuego solitario (RAMÍREZ MACÍAS, 2011), pasando a existir comunidades activas de jugadores que consumen este producto de ocio electrónico con otros jugadores de manera simultánea (CARRILLO, 2015).

El fenómeno de los deportes electrónicos, más conocidos mundialmente como e-sports (SEGAL, 2014; WAGNER, 2006), se ha convertido hoy en día en un elemento fundamental de la cultura y el ocio digital entre los jóvenes, siendo la industria del videojuego el sector cultural y de entretenimiento que más recauda en multitud de países. Este concepto surge de la profesionalización del mundo competitivo de los videojuegos, lo que supone una reinterpretación del modo de consumo de éstos y de su naturaleza lúdica, pasando a formarse un espectáculo deportivo donde el videojuego es el principal agente junto a los jugadores (CARRILLO, 2016). Los eventos de esta índole han tomado prestados elementos de los deportes tradicionales (narradores, analistas, estadistas, instalaciones, estructuras de liga, temporalización) que dotan, por un lado, de comodidad y seguridad al público con estructuras ya conocidas y, por otro, de espectacularidad a esta nueva forma de competición. Además se añaden otros elementos que dotan de identidad y enriquecen a este espectáculo de masas, como son la interacción del público con la propia retransmisión y los narradores, los componentes tecnológicos o el cuidado de todos los aspectos audiovisuales (CARRILLO, 2015; HILVERT-BRUCE, NEILL, SJOBLOM; HAMARI, 2018).

En este artículo, y de ahí esta introducción, se aborda la posibilidad de inclusión de los e-sports dentro del concepto deporte, pues, aunque el debate ya quedó establecido hace años (JENNY; MANNING; KEIPER; OLRICH, 2016), hoy día existe una candente discusión acerca de si los e-sports pueden considerarse y/o denominarse deporte (JENNY; MANNING; KEIPER; OLRICH, 2016; PARRY, 2018; SÁNCHEZ; DAVIS REMILLLARD, 2018). Al respecto, sin duda hay que subrayar que el propio Comité Olímpico Internacional (C.O.I) ha estado realizando acciones de acercamiento a este ámbito, estudiando la posibilidad de incluirlos en los próximos Juegos Olímpicos de 2020 (CLAPPERTON, 2015; JENNY; MANNING; KEIPER; OLRICH, 2016; KATES, 2015). Este acercamiento del C.O.I a los e-sports no atiende ni a la cercanía de éstos con el ideario olímpico, ni siquiera con su posible inclusión a nivel semántico dentro del concepto deporte. Más bien, el interés del C.O.I radica en un plano económico, pues las ganancias del sector de los e-sports son muy destacables, y estratégico, puesto que el número de practicantes, e incluso de seguidores de estos eventos, es cada vez mayor. Por tanto, con este acercamiento el C.O.I, institución hegemónica del deporte a nivel mundial, se aviene positivamente con un sector tremendamente pujante, incluso se postula para tutorizar sus competiciones en el máximo escaparate deportivo a nivel planetario: los Juegos Olímpicos. Este posicionamiento no tiene porque ser negativo en sí mismo, ya que, 
como sucedió con la aprobación de la Carta Europea del Deporte de 1992, donde se optó por una definición amplia del concepto deporte, con idea de que las políticas deportivas beneficiaran a la mayoría de la ciudadanía; en este caso puede ser positivo ampliar de facto el campo semántico del concepto deporte, incluyendo dentro de él a los e-sports, pues ello puede beneficiar a todos sus practicantes y seguidores, al incluirlos en un marco institucional que les proteja.

En todo caso, este debate sobre el carácter deportivo de los e-sports no deja indiferente a nadie, ya que supone una desestabilización del sistema deportivo y pone en jaque todo el ideario preconcebido alrededor de la idea de deporte, la concepción del deporte y otras cuestiones como las actividades y modalidades que entrarían dentro de este concepto. Wagner (2006), primer autor en dar una definición reconocida del fenómeno (y cercana al ámbito deportivo), describe los e-sports como un área de las actividades deportivas donde los participantes desarrollan y entrenan capacidades mentales y físicas en el uso de las TIC. Actualmente, tras una pronta y necesaria mejora, el término ha evolucionado hasta matizar que los deportes electrónicos se fundamentan en el uso de videojuegos con fines competitivos como vehículo competitivo a través de los que se obtienen recompensas económicas u otras como premio por alcanzar los primeros lugares en las competiciones, ligas y eventos (MARCANO, 2012). En cualquier caso, como ya se ha comentado, es un fenómeno en constante cambio y evolución, por lo que habrá que seguir avanzando para encontrar una correcta definición de los e-sports que englobe y recoja todo el corpus de actividades que entrarían dentro de este concepto (JENNY; MANNING; KEIPER; OLRICH, 2016; JIN, 2010).

Además de esta definición de e-sport, en base al constante debate acerca de la palabra que mejor recoge a este nuevo ámbito, tanto en el propio fenómeno como en la literatura científica se ha pasado por conceptos como e-sport, E-Sport, e-Sport, eSport y otros sucedáneos. Actualmente, el debate ya no es tal dentro de los agentes y público de nicho, pues se ha acuñado esport o esports por su frecuencia, simplicidad, usabilidad y otras razones lingüísticas similares a lo ocurrido con la palabra email, pero aún no ha entrado directamente en el ámbito investigador debido a la confusión que crea en diferentes idiomas, así como que, en la escasa producción científica existente, se ha ido usando e-sport como descriptor y/o keyword. Donde si existe consenso es el uso de la $\mathrm{E}$ mayúscula en esta palabra al usarse al comienzo de oración.

Finalmente, hay que indicar que, de acuerdo con el objetivo de este ensayo, en los siguientes apartados se realizará un análisis crítico del concepto de deporte, así como de los argumentos tanto a favor como en contra acerca de la inclusión de los e-sports dentro de este concepto.

\section{EL TÉRMINO DEPORTE}

La definición del término deporte encuentra en el ámbito de los Juegos Olímpicos una de sus fuentes más relevantes debido a que son considerados comúnmente como el súmmum de la competición deportiva. Sin embargo, el deporte 
no solo es practicado por los deportistas olímpicos, sino que forma parte del tejido social como fenómeno humano y global que es, lo que genera una necesidad de permeabilidad del término a todos los niveles. La revisión terminológica acerca del concepto deporte, aporta numerosas definiciones que apuntan a diferentes elementos, por lo que el concepto está reconfigurando constantemente para acotar las diferentes actividades que se practican y no a la inversa, siendo las actividades quienes tengan que incorporarse conceptualmente al término (PUIG; HEINEMANN, 1991).

Existen ocasiones en que el uso del término deporte se realiza con connotaciones que no son las habituales, ya que la sociedad usa el significante 'deporte' para referirse a multitud de actividades, muchas veces confundiéndose con el ejercicio o la actividad física (PARRY, 2018). Tanto es así que la propia definición de deporte que figuran en los diccionarios lo clasifican como 'diversión', 'recreación', 'diversión' (así como los verbos de estos sustantivos) o, el caso más discutido por los académicos, los términos 'juego' o ‘jugar'. No obstante, un hecho común en la mayoría de las definiciones del término deporte es la inclusión de varios rasgos (GUTTMANN, 1978; JENNY; MANNING; KEIPER; OLRICH, 2016; PARRY, 2018; SUITS, 2007):

a) La institucionalización, es decir, la existencia de organismos nacionales e internacionales que lo administren, lo regulen, etc.

b) La posesión de un conjunto de reglas fijas y comunes.

c) El carácter motriz y/o un conjunto de habilidades físicas propias.

Aunque estos rasgos suelen ser comunes en todas las definiciones, existen otras características relevantes como el carácter humanístico, el competitivo o el componente ético y moral de las prácticas deportivas; provenientes todas ellas de definiciones más cercanas a los campos de la sociología del deporte (GUTTMANN, 1978) o la filosofía del deporte (SUITS, 2007).

Todas estas características, a la vez que proponen la definición del término, actúan como criterios de exclusión para delimitar qué es deporte y que no lo es. Por consiguiente, podremos utilizar cada característica para analizar cualquier modalidad deportiva, disciplina o competición, incluida los e-sports; incluso, se pondría de manifiesto si esta concepción actual del término es correcta para delimitar, desde un paradigma platónico, su eidos.

Para cerrar este punto, es importante clarificar que el concepto de deporte que se analiza se define en el tiempo y el espacio actual (PARRY, 2018), es decir, la definición de deporte no puede remitirse a las prácticas inglesas del siglo XIX, ni tampoco al deporte practicado en España en la década de 1940; es necesaria una conceptualización del término en y para la sociedad actual, que no rechace concepciones anteriores pero que dé respuestas a lo que hoy día es el deporte.

\subsection{INSTITUCIONALIZACIÓN}

La institucionalización es el proceso mediante el cual se crean o fundan diferentes organismos que dirigen y/o regulan una actividad (en este caso deportiva) tanto nacional como internacionalmente. 
El argumentario sobre incluir esta característica en la definición del deporte proviene de la idea de que se necesita de un ente externo al propio juego que regule el mismo, haciendo que las reglas que lo conforman se cumplan y sean estables (DREWE, 2003; SUITS, 2007; TAMBURRINI, 2000). Además, otro argumento recae en que, si un juego alcanza la representación suficiente por parte de organismos nacionales e internacionales, eso significa que ya es suficientemente relevante como para considerarlo como un deporte, ya que posee interés social, histórico y cultural (SUITS, 2007).

Consideramos que es un argumento con limitaciones plantear que un juego es deporte solo porque un organismo externo así lo defiende, en vez de ahondar en las características intrínsecas de esa actividad que la definen como deporte. Se trata de imponer un criterio externo porque a nivel interno los límites entre lo que es juego y lo que es deporte no están claros, por ello, en vez de ahondar en el esfuerzo intelectual que permita una delimitación clara y precisa de ambos términos (si es que la hay), se opta porque haya una institución que sancione qué es deporte y qué no lo es. Algunas de estas características intrínsecas a las que podría atenderse son las acuñadas por el sujeto, ya que el mismo es el origen y creador de estas actividades, así como otras como las sensaciones, emociones, valores e implicaciones morales/ éticas que le supone al ser humano esta actividad al ser partícipe de ella.

Esta situación de debilidad argumentativa provoca no pocas excepciones. Por poner un ejemplo, el ajedrez es aceptado como deporte por el C.O.I, a pesar de la ausencia de motricidad, mientras que las damas no, bajo el argumento de que no está suficientemente institucionalizado. No obstante, ha sido la propia federación de ajedrez la que ha intentado incluir esta actividad en sus organismos sin éxito alguno. Por tanto, las damas se han intentado institucionalizar, pero como este propósito no ha sido logrado, no son un deporte como sí lo es el ajedrez.

Por otro lado, deportes colectivos con más trayectoria histórica en Juegos Olímpicos, como el balonmano, el voleibol o el hockey, han aceptado dentro de sus federaciones a otras actividades que, hasta que no fueron aceptadas por estas instituciones, no pasaron a ser consideradas deporte; como por ejemplo el balonmano playa, el voleibol playa o el hockey subacuático. Todo ello no hace sino incidir en la arbitrariedad que tiene esta institución, hegemónica a nivel planetario, con respecto a fundamentar qué actividades entran en el listado de deportes que reconoce.

Parlebas $(1986,2001,2003)$ defiende en su definición de deporte que, basándose en un criterio sociológico (y externo), los juegos deportivos serán o no deporte dependiendo de la institucionalización. En esta misma clasificación, incluía a los videojuegos, defiendo la idea de que no pueden ser deporte solo por no contar con ese respaldo institucional.

Al respecto, hay que subrayar a modo de aclaración, que si bien no todos los juegos son deporte, sí son juegos todos los deportes (COAKLEY, 2008; GUTTMANN, 1978). Una de las diferencias entre juego y deporte, afirma Zapico en el año 2003 (citado de RAMíREZ, 2006), es la búsqueda, entre otros factores, del rendimiento. En el ámbito de los videojuegos ocurre exactamente eso. Todos los videojuegos no son e-sports, aunque si todos los e-sports son videojuegos. La diferencia proviene, 
grosso modo, del carácter competitivo de éstos, así como de la reglamentación propia de los e-sports (COAKLEY, 2008; HEMPHILL, 2005).

Según esta definición de Parlebas $(2001,2003)$, si los e-sports se institucionalizasen, serían inmediatamente considerados deporte, sin fijarnos en el resto de las características ya que éstas estarían cumplidas.

En cualquier caso, la institucionalización de los e-sport ya es un hecho. De manera similar a lo que ocurre en el deporte tradicional, existen organismos, tanto locales y regionales como nacionales e internacionales que están regulando el sector: clubes, federaciones, asociaciones de clubes, etc. (BOROWY; JIN, 2013; KOW; YOUNG, 2013; TAYLOR, 2012; WITKOWSKI, 2012). Estas instituciones no solo organizan y reglamentan las competiciones, sino que tratan de defender los intereses de los jugadores, de hecho, muchas de ellas, propugnan la creación de una legislación que respalde a todos los entes que intervienen en el sector. En definitiva, tienen como finalidad regular y organizar esta actividad.

\subsection{REGLAMENTACIÓN}

La reglamentación es un proceso mediante el cual se confeccionan y definen el conjunto de normas referentes a una actividad. No solo se basan en la permisión y/u obligación de determinadas acciones, sino que organiza y describe cómo es el juego, las características del mismo y otros aspectos intrínsecos al mismo (GUTTMANN, 1978; SUITS, 2007).

Para que un juego sea considerado deporte no solo debe tener reglamentación, sino que ésta debe poseer elementos fijos que sirvan de cimentación para que esta actividad se realice de forma muy similar en todos los lugares del mundo. Además, se busca que estas normas de juego hayan tenido tanto desarrollo como para producir un ecosistema de juego complejo, con habilidades o recursos propios del mismo (PARLEBAS, 2001). Es, por ejemplo, lo que ocurre en numerosos juegos de pelota, considerados muchos de ellos juegos tradicionales. Algunos tienen elementos comunes, pero no son suficientes para catalogarlos como un mismo deporte, ya que las reglas de cada juego cambian incluso dentro de una misma zona geográfica. Por el contrario, si un atleta realiza la prueba de 100 metros lisos en los campeonatos del mundo o en el campeonato nacional de su país, estaría practicando un deporte, ya que está bajo una reglamentación dada y común con independencia del contexto. Sin embargo, no se aplicarían estas reglas al mismo atleta entrenando en un pabellón, por lo que, en este caso, no estaríamos hablando de deporte sino de actividad física.

Ocurriría parecido en un hipotético partido de fútbol con amigos, donde estaríamos ante un deporte o un juego dependiendo exclusivamente de la modificación de los elementos constitutivos de la actividad: tiempo reglamentario, número de jugadores, sanciones disciplinarias, etc. De esta forma, vemos como la reglamentación o la ausencia de ésta, condiciona totalmente la actividad.

En el sector de los e-sports ocurre algo similar. Los diseñadores de los videojuegos, a la vez que creadores de los mismos son sus legisladores, ya que limitan, en mayor o menor medida, las acciones que pueden realizar o no los 
jugadores en cada uno de estos videojuegos (GÓMEZ, 2007). Sin embargo, también pueden diseñarse videojuegos donde exista un marco de acciones amplio y que sea el mismo jugador el que delimite qué se puede y qué no se puede hacer para conseguir el objetivo. De hecho, incluso el objetivo del juego puede ser marcado por el jugador (TAYLOR; WITKOWSKI, 2010; TAYLOR, 2012; WITKOWSKI, 2012). Llevado a la competición, el organismo encargado de auspiciarla puede delimitar el conjunto de situaciones válidas, existiendo una reglamentación propia para cada torneo, así como árbitros y/o jueces que respaldan la integridad competitiva de estos eventos. Asimismo, según Carrillo (2016):

Los encuentros deportivos tienen lugar bajo la aceptación, por parte de los contendientes, de unas normas determinadas e igualitarias a las que se someten: las reglas del juego. Esta reglamentación ofrece un marco sobre el que se desarrolla la competencia en unas condiciones equitativas, pues son conocidas de antemano por los deportistas (y por el público) e impide la ventaja inicial de los participantes, lo que deja la resolución de los enfrentamientos en manos del talento, la habilidad o la pericia en la práctica del deporte dentro de ese marco normativo (CARRILLO, 2016, p. 8).

Tanto es así que cada videojuego dentro de los e-sports se basa en una reglamentación (JENNY; MANNING; KEIPER; OLRICH, 2016) que dicta las normas del mismo a nivel internacional, ya que la versión del videojuego será la misma para todos los usuarios a nivel mundial. Incluso, tal y como ocurre con los deportes tradicionales, estas reglas están sujetas a evolución en base a las experiencias propias de la competición, de hecho, van modificándose buscando espectacularidad, integridad competitiva, igualdad de condiciones para los jugadores, etc.

\subsection{CARÁCTER MOTRIZ}

En primer lugar, este apartado requiere delimitar que es una acción motriz o motricidad. Este concepto proviene del movimiento generado por el sistema locomotor, existiendo diferentes tipos de motricidad (gruesa y fina) y etapas de motricidad (ganglionar, cerebral, espinal, piojosa, muscular y articular). El nivel de precisión del movimiento (JENNY; MANNING; KEIPER; OLRICH, 2016) así como los músculos implicados (HAIBACH; REID; COLLIER, 2011) diferencian las habilidades motoras en gruesas (realizar un pase) o finas (manipular un objeto). Sin embargo, el término motricidad es mucho más profundo, conteniendo aspectos epistemológicos como la pertenencia al propio ser. Así, la motricidad requiere de los procesos y las funciones del organismo y el control psíquico que cada movimiento trae consigo. Por lo tanto, la motricidad no solo representa esa parte visible, sino la parte interna del movimiento. Esto supondría, en primera instancia y de manera superficial, que toda aquella actividad donde un individuo realice movimientos, que esté reglada e institucionalizada, podría considerarse deporte. Sin embargo, existen actividades, consideradas deporte, donde el sujeto no realiza la acción, como la colombofilia. Con ejemplos como éste, donde el sujeto no interviene, vemos como la definición del deporte en base a la motricidad implicada en la actividad no es totalmente ajustada a todas las actividades que hoy día son consideradas como deporte.

Algunos teóricos, como Parry (2018), defienden que no solo es necesario el movimiento, sino que debe ser una acción física donde los movimientos reales sean 
relevantes en el juego (FREEMAN; WOHN, 2017). Ese es uno de los argumentos de los detractores del ajedrez como deporte, ya que defienden que quién haga el movimiento o cómo lo haga es irrelevante para el resultado del juego; lo importante es dónde acaba la pieza (OUSTERHOUDT, 1977). En cualquier caso, volvemos a toparnos con una inconsistencia del concepto deporte, pues el ajedrez es considerado como uno de ellos, aunque los movimientos implicados en este juego no son significativos para el resultado del mismo.

Por otro lado, existen numerosas actividades donde los movimientos del sujeto practicante forman parte de la acción deportiva, pero no son determinantes para el resultado de la actividad. Por ejemplo, en equitación los movimientos significativos para lograr el éxito en esa actividad deportiva los realiza el caballo, las acciones del jinete son secundarias. Es decir, sin restar valor al hecho de que el jinete ayuda y guía al caballo, sin duda es éste último el que realiza la acción motriz significativa.

Por otro lado, existen autores que argumentan que el deporte no es solo el movimiento que se realiza, sino el control del no-movimiento o control postural (PARRY, 2018), así como el espectro de percepciones y decisiones que se toman en este transcurso temporal (FREEMAN; WOHN, 2017). Así, desde la aceptación del ajedrez como deporte, nos hemos encontrado con otro caso particular que ha buscado este reconocimiento: los juegos de cartas. Actividades como el póker o el bridge, reguladas e institucionalizadas, han luchado por ser incluidos dentro del deporte, aduciendo su valor deportivo en base a la argumentación anterior. Al respecto, es llamativo que el principal escollo para la consideración de estos juegos de cartas como deportes radica en que poseen un factor de azar o varianza muy alto, que hace que la habilidad pase a un segundo plano (SUITS, 2007). Por tanto, no es su falta de motricidad el principal problema, pues el ajedrez está al mismo nivel, sino la gran relevancia que el azar tiene en estos juegos.

Quizás, para delimitar más correctamente el tipo de motricidad propia de los deportes, se debería concretar qué naturaleza tendrían estas acciones motrices. Hay autores que hablan de diferenciar entre habilidades motrices básicas (lanzamientos, recepciones, giros, saltos, desplazamientos) y/o genéricas (golpeos, impactos, fintas, pases, dejadas, ...). Otros optan por la cuantificación de la carga, a través de la frecuencia cardiaca o el volumen de oxígeno máximo (MITCHELL; HASKELL; SNELL; VAN CAMP, 2005), incluso los hay que optan por escalas subjetivas como la de Borg. Sin embargo, actualmente, no pueden considerarse como delimitadores fiables de la motricidad propia del deporte a ninguna de las opciones anteriores, ya que existen factores internos y externos a las diferentes prácticas que pueden condicionar el tipo de acción y la intensidad con la que se realiza, sin que ello implique que en unos casos se está practicando deporte y en otros no.

Hasta que no se defina con precisión el tipo de motricidad propia del deporte, si es que realmente ese es el camino para dilucidar qué es deporte, los e-sports pueden considerarse deporte ya que en ellos se registran patrones motrices finos, reflejos rápidos, gran destreza manual y excelente coordinación mano-ojo (RAMBUSCH; JAKOBSSON; PARGAN, 2007), utilizando para ello un implemento como lo hacen otros muchos deportes. Así es posible comparar a los e-sport con otros deportes (tiro 
con arco, tiro olímpico, billar, dardos), incluso muchos de ellos olímpicos. En cualquier caso, estas actividades, en su máximo rendimiento, suponen altas implicaciones de los mecanismos de percepción y decisión (GRANIC; LOBEL; ENGELS, 2014; HEMPHILL, 2005; KRETCHMAR, 2005; TORIL; REALES; BALLESTEROS, 2014; WAGNER, 2006), siendo estos dos los factores clave de rendimiento, como lo son también en el rendimiento de multitud de deportes consolidados.

Ampliando todo lo expuesto, la realidad virtual y los exergames (STAIANO; CALVERT, 2011) forman parte del ámbito de los videojuegos, donde ya la actividad física se torna a movimientos o acciones motrices gruesas en las que se compromete todo el cuerpo y/o grandes grupos musculares. Bajo este argumentario, no habría entonces dudas sobre que los e-sports poseen una motricidad propia del ámbito deportivo.

\subsection{OTRAS CARACTERÍSTICAS}

Tal y como se adelantó en la introducción, desde la perspectiva de la sociología del deporte y de la filosofía del deporte, existen una serie de características del concepto deporte que vienen a completar la actual delimitación del término.

\subsection{CARÁCTER HUMANÍSTICO}

Uno de estos rasgos del deporte es su carácter humanístico, ya que, aunque multitud de seres vivos incluyen el juego en sus actividades, somos los únicos seres vivos capaces de generar deportes, si bien es cierto que existen modalidades deportivas donde diferentes animales participan o se ven involucrados, siempre lo hacen bajo subordinación al ser humano (PARRY, 2018). Lo mismo ocurriría con el uso de ciertos implementos, máquinas y/o tecnología, siendo parte del deporte, pero siempre bajo el control y uso humano.

Pasando a la ejemplificación, la equitación es considerada un deporte olímpico dado que el ser humano controla en todo momento al equino, en contraposición a las carreras de perros u otros seres vivos, que no son considerados deporte debido a que estos no son controlados por el ser humano al ser soltados. Por esta regla, actividades como la colombofilia o la caza deportiva no deberían ser consideradas deporte, aunque lo son. Estamos de nuevo ante otro caso de intrusismo, debido a la pobre delimitación que existe actualmente del concepto deporte.

Si pasamos a valorar los deportes donde se incluye un motor, la mayoría de estos son considerados deporte, aunque no forman parte de los Juegos Olímpicos. Esto es debido a que, según comentan los expertos, aunque el vehículo es controlado totalmente por el sujeto, no puede realizarse una atribución causal de que el ganador sea el mejor piloto ya que podría tener mejor vehículo que sus competidores y deja a un lado ese rasgo totalmente humanista. Por otro lado, la vela si es deporte olímpico, aunque ocurre algo similar. En cualquier caso, de nuevo no parece un rasgo que delimite con precisión el término, por lo que actualmente no puede tomarse como sesgo absoluto.

Los detractores de los e-sports, bajo este prisma comentan que, al igual que las carreras por control remoto, las peleas de robots y sucedáneos, al ser controlado 
otro ente mediante radiofrecuencia $u$ otro medio, sin que haya contacto directo, no pueden considerarse como deporte, porque se aleja de este humanismo. Nos tendríamos que preguntar, en este punto, quién es el atleta, si el avatar mediante el que se juega o el propio jugador y si éste es un rasgo suficiente para su exclusión. Autores como Sánchez y Martinez (2014) o Sánchez y Davis (2018) defienden que no, ya que comentan que los e-sports humanizan el deporte haciéndolo accesible para todos, siendo un proceso de democratización, humanización y universalización de la experiencia deportiva, pero sin alienarnos y siendo parte de la realidad empírica, ya que mejoran nuestra coordinación, capacidades motoras y capacidades espaciotemporales (SÁNCHEZ; DAVIS, 2018).

\subsection{COMPETICIÓN}

Esta característica se refiere al carácter competitivo del deporte (GUTTMANN, 1978). Quizás éste sea el rasgo donde más consenso a favor pueda existir, pero eso no lo deja exento de algunas críticas. El primer matiz a subrayar es que solo se considera deporte aquellas actividades donde la competición se realice contra otro competidor (DREWE, 2003) o grupo de competidores, nunca con uno mismo, de ahí que sea un factor intrínseco al deporte la autosuperación (JEU, 1972). El sujeto solo puede vencer a otros por medio de una dominación de sí mismo, sirviendo el oponente como camino para lograr ir más allá de sí, más allá de lo que es, alcanzando esa autosuperación. El segundo matiz importante es que hay que hacer una distinción entre competición y concurso. La danza, se puede disfrutar bajo diferentes modos: ritual, recreativo, etc., pero también como una forma de competición. Sin embargo, ésta no está contemplada como deporte, debido a su fuerte carácter artístico y subjetivo, por lo que reglarla, además de ser una ardua tarea, encasillaría al mismo y perdería mucha libertad artística. Por el contrario, la natación sincronizada posee también grandes dosis de ese carácter artístico, pero es un deporte. Por tanto, estamos de nuevo ante una deficiente acotación conceptual del término deporte.

Con respecto a los e-sports el componente competitivo es un rasgo primario e intrínseco a su propia definición (WAGNER, 2006), ya que un videojuego se considera e-sports, entre otras cosas, por este rasgo competitivo. Por tanto, bajo esta característica, los e-sport por supuesto que son deporte, incluso antes que otros deportes en los que el factor competitivo no está tan enraizado, como es el caso de la gimnasia acrobática donde, sin duda, hay competición, pero no es tan esencial para su práctica como sí lo es en el caso de los e-sport, puesto que al jugar siempre compites contra otro/s.

\section{7 ÉTICA Y MORALIDAD}

El último rasgo, muy discutido por los filósofos del deporte, es el carácter ético y/o moral que debe poseer el deporte. Además de este debate sobre la concepción de los e-sports como deporte, estas últimas décadas se ha discutido sobre si el deporte era un transmisor de valores de manera intrínseca y directa y, en el caso de que lo fuera, si eran positivos y/o negativos (GONZÁLEZ, 2013). Existen argumentos desde todas las posiciones, aunque la corriente más arraigada es aquella que defiende que 
el deporte, al ser una construcción humanística y social, puede ser un trasmisor de valores, aunque no significa que éstos sean positivos de manera inequívoca.

Los estudios actuales sobre filosofía del deporte marcan que el deporte debe tener un marcado corte ético (GONZÁLEZ, 2013), lo que podría ser un rasgo diferencial para delimitar qué es deporte y que no lo es.

Por ejemplo, los munus gladiatoris poseían todos los elementos que marcan al deporte actual: reglamentación, actividad motriz, competición, institucionalización (el estado romano los organizaba), e, incluso existían apuestas, métodos de entrenamiento y entrenadores especializados. De esta forma, poseerían todos los elementos que existen en numerosos deportes consagrados como el fútbol o el atletismo. Sin embargo, la diferencia la marca el componente ético: en esta actividad existen daños graves, extremos e incluso muertes. Ahora bien, también existen actividades como las artes marciales mixtas, el kickboxing, el muai thay u otros deportes de combate similares que son considerados como deportes. En éstos existen lesiones irreversibles, además de ser extremadamente violentos e incluso, algunos de ellos, son practicados por niños de menos de 12 años. ¿Acaso hay una radical diferencia entre los gladiadores romanos y estos deportes del siglo XXI?

Por el contrario, actividades como el paintball o el airsoft están siendo consideradas fuera del concepto deporte por, supuestamente, sobrepasar esos límites éticos, ya que utilizan réplicas de armas reales o proyectiles de plástico, además de que las competiciones simulan prácticas bélicas ya sea con la vestimenta, espacios y/u otros elementos del contexto. Sin embargo, el riesgo de lesiones graves, violencia extrema o que sean practicadas por menores de 12 años es significativamente menor que en el caso de algunas artes marciales mixtas.

Con respecto a esta dimensión ética, hay que subrayar que, tal y como se indicó al principio de este ensayo, el C.O.I ha estado muy interesado en incluir los e-sports en los próximos Juegos Olímpicos (CLAPPERTON, 2015; KATES, 2015). Tanto es así que se ha realizado varias pruebas al respecto, incluyéndolos en los últimos Juegos Asiáticos y en los próximos Juegos de Invierno. No obstante, en la segunda mitad del 2018, este organismo ha realizado unas declaraciones donde duda sobre la incorporación de los e-sports a los Juegos Olímpicos, ya que algunos de ellos tienen cierto componente violento y pueden promover actitudes violentas, las cuales serían contrarias al espíritu olímpico. También añaden que, en determinados e-sports, existe un lenguaje donde se usa frecuentemente las palabras 'matar', 'capturar', 'eliminar' y verbos o sustantivos similares.

Sin embargo, la argumentación anterior es bastante débil, pues dentro de los propios Juegos Olímpicos existen deportes que tienen cierto componente violento, como el boxeo o el pentatlón moderno. ¿Éstos no sobrepasan los límites éticos del espíritu olímpico? En cualquier caso, no todos los e-sports tienen un cariz violento. Existen múltiples e-sports, de forma que cada uno de ellos supone una modalidad deportiva única e independiente, lo que debe ocasionar un análisis individualizado sobre cuáles de ellas podrían formar parte del programa olímpico. 


\section{CONCLUSIONES: HACIA UNA NUEVA DEFINICIÓN DEL DEPORTE}

Si bien el concepto deporte posee tres características ampliamente aceptadas como definitorias del mismo, la realidad nos muestra cierta arbitrariedad con respecto a la consideración o no de muchas actividades dentro del concepto deporte. hecho de que existan varias excepciones que, sin cumplir con las tres características definitorias, sean consideradas como deporte, no deja de ser una llamada de atención sobre la necesidad de poner a prueba o cuestionar la definición actual de deporte, ya que, como hemos subrayado, el marco delimitado por esta definición es abstracto, subjetivo y, en numerosas ocasiones, arbitrario.

Los diferentes epígrafes de este ensayo no buscaban, al menos de forma directa, defender que, a tenor de la actual definición del concepto deporte, los e-sports deberían ser considerados como deportes e incluso dentro del programa olímpico. No tratamos de hacer una apología de los e-sports, tan solo hacer un análisis crítico de lo que hoy día se considera como deporte, utilizando como piedra angular de este análisis a los e-sports. Así, se han presentado varios argumentos que inciden en la poca firmeza, claridad y precisión del concepto deporte. Consideramos que es necesario realizar una reflexión y un gran esfuerzo intelectual acerca del concepto deporte y su delimitación semántica, pues es fundamental hallar los cimientos definitorios de lo que es deporte en la realidad actual del siglo XXI. De forma que se pueda delimitar con precisión que actividades caen dentro de este concepto y cuáles no.

Sin embargo, si seguimos considerando al deporte como un objeto externo al sujeto, como una realidad independiente de éste, seguiremos fallando en nuestros intentos, de forma que los límites de qué es deporte seguirán difusos y actividades como el ajedrez o la colombofilia seguirán siendo deporte y, sin embargo, cualquier juego tradicional con un marcado carácter motriz y perfectamente reglamentado, como el balón prisionero, no lo será. El deporte tiene su esencia en el ser humano, por lo que su estudio fenomenológico no puede estar desligado del sujeto, sino focalizado en él. Así, podría iniciarse este esfuerzo definitorio del concepto a partir de describir las vivencias fenomenológicas acontecidas en el deporte, para encontrar rasgos comunes en todas ellas, lo que nos permitiría definir qué es la experiencia deportiva, ya no habría un deporte ideal, externo al ser humano y que existe con independencia de éste, sino un deporte vivido que acontece en la propia experiencia vital y solamente en ella.

\section{REFERENCIAS}

BOROWY, Michael; JIN, Dal Yong. Pioneering eSport: The Experience Economy and the Marketing of Early 1980s Arcade Gaming Contests. International Journal of Communication, v. 7, n. 21, p. 2254-2274, 2013. 
CARRILLO, José Agustín. From players to viewers: the construction of the media spectacle in the e-sports context. Anàlisi, n. 55, p. 1-16, 2016. DOI: https://doi.org/10.7238/a.

v0i55.2893

CARRILLO, José Agustín. La dimensión social de los videojuegos 'online': de las

comunidades de jugadores a los 'e-sports'. Index. Comunicación, v.1, n.5, p. 39-51, 2015.

CLAPPERTON, Guy. The Debate: For \& against e-sports. Engineering \& Technology, v. 10, n. 1, p. 28b, 2015. DOI: https://doi.org/10.1049/et.2015.0348

COAKLEY, Jay. Sports in society: Issues and controversies. $10^{\text {th }}$ ed. New York, NY: McGraw-Hill, 2008.

DREWE, Sheryle. Why sport? Toronto: Thompson, 2003.

FREEMAN, Guo; WOHN, Donghee Yvette. eSports as An Emerging Research Context at $\mathrm{CHI}$. In: Proceedings of the $2017 \mathrm{CHI}$ Conference Extended Abstracts on Human Factors in Computing Systems - CHI EA '17. New York: ACM, 2017. p.1601-1608. DOI: https://doi.org/10.1145/3027063.3053158

GÓMEZ, Salvador. 'Videojuegos: el desafío de un nuevo medio a la comunicación social'. Historia y Comunicación Social, v.12, p. 71-82, 2007.

GONZÁLEZ, Javier Durán. Ética de la competición deportiva: valores y contravalores del deporte competitivo. Materiales para la Historia del Deporte, n. 11, p. 8-27, 2013.

GRANIC, Isabela; LOBEL, Adam; ENGELS, Rutger. The benefits of playing video games. American Psychologist, v. 69, n. 1, p. 66-78, 2014. DOI: https://doi.org/10.1037/ $\underline{a 0034857}$

GUTTMANN, Allen. From ritual to record: The nature of modern sports. New York, NY: Columbia University, 1978.

HAIBACH, Pamela; REID, Greg; COLLIER, Douglas. Motor learning and development. Champaign, IL: Human Kinetics, 2011.

HEMPHILL, Dennis. Cybersport. Journal of the Philosophy of Sport, v. 32, n. 2, p. 195207, 2005. DOI: https://doi.org/10.1080/ 00948705.2005.9714682

HILVERT-BRUCE, Zorah.; NEILL, James. T.; SJÖBLOM, Max.; HAMARI, Juho. Social motivations of live-streaming viewer engagement on Twitch. Computers in Human Behavior, 84, 58-67, 2018. DOI: https://doi.org/10.1016/j.chb.2018.02.013

JENNY, Seth; MANNING, R. Douglas; KEIPER, Margaret; OLRICH, Tracy. Virtual(ly) Athletes: Where eSports Fit Within the Definition of "Sport". Quest, v. 69, n. 1, 2016. DOI: https://doi.org/10.1080/00336297.2016.1144517

JEU, Bernard. What is sport? Diogenes, v. 20, n. 80, p. 150-163, 1972. . DOI: https://doi. org/10.1177/039219217202008007

JIN, Dal Yong. Esports and television business in the digital economy. In: JIN, Dal Yong (ed.). Korea's online gaming empire. Cambridge, MA: MIT Press, 2010. p. 59-79. DOI: https:// doi.org/10.7551/mitpress/9780262014762.001.0001

KATES, Andrea. The Debate: For \& against e-sports. Engineering \& Technology, v. 10, n. 1, p. 28a, 2015. DOI:10.1049/et.2015.0348 Disponível em: https://ieeexplore.ieee.org/stamp/ stamp.jsp?tp=\&arnumber=7084541. Acesso em: 14 jun. 2020. 
KOW, Yong Ming; YOUNG, Timothy. Media technologies and learning in the starcraft esport community. In: Proceedings of the 2013 Conference on Computer supported cooperative work (CSCW '13). New York: ACM, 2013. p. 387-398. DOI: https://doi. org/10.1145/2441776.2441821

KRETCHMAR, R. Scott. Practical philosophy of sport and physical activity. $2^{\text {nd }}$ ed. Champaign: Human Kinetics, 2005.

LAFRANCE, Jean Paul. El juego interactivo: el primer medio de masas de la era electrónica. Quaderns del CAC, n. 15, p. 59-68, 2003.

MARCANO, Beatriz. Características sociológicas de videojugadores online y el e-sport. El caso de Call of Duty. Pedagogía Social. Revista Interuniversitaria, n. 19, p. 113-124, 2012. DOI: https://doi.org/10.7179/PSRI_2012.19.07

MITCHELL, Jere H.; HASKELL, William; SNELL, Peter; VAN CAMP, Steven. Task Force 8: classification of sports. Journal of the American College of Cardiology, v. 45, n. 8, p. 1364-1367, 2005. DOI: https://doi.org/10.1016/j.jacc.2005.02.015

OUSTERHOUDT, Robert. The term "sport": Some thoughts on a proper name. International Journal of Physical Education, v.14, n. 2, p. 11-16, 1977.

PARLEBAS, Pierre. Elementos de Sociología del Deporte. Cádiz: Unisport, 1986.

PARLEBAS, Pierre. Elementos de Sociología del Deporte. Málaga: Unisport, 2003.

PARLEBAS, Pierre. Juegos, deporte y sociedad. Léxico comentado en praxeología motriz. Barcelona: Paidotribo, 2001.

PARRY, Jim. E-sports are Not Sports. Sport, Ethics and Philosophy, v. 13, n. 1, p. 3-18, 2018. DOI: https://doi.org/10.1080/17511321.2018.1489419

PUIG, Nuria; HEINEMANN, Klaus. El deporte en la perspectiva del año 2000. Papers: Revista de Sociología, n. 38, p. 123-141, 1991. DOI: https://doi.org/10.5565/rev/papers/ $\underline{\mathrm{v} 38 \mathrm{n} 0.1613}$

RAMBUSCH, Jana; JAKOBSSON, Peter; PARGMAN, Daniel. Exploring E-sports: A case study of gameplay in Counter-Strike. In: Situated play : The 2007 world conference of Digital Games Research Association [Internet]. Digital Games Research Association (DiGRA), 2007. p. 157-64. Disponível em: http://www.diva-portal.org/smash/get/ diva2:25495/FULLTEXT01.pdf Acesso em: 14 Jun. 2020.

RAMÍREZ MACÍAS, Gonzalo. Deporte vs. juego. A la búsqueda de un concepto integrador. Efdeportes.com, ano 10, n. 94, 2006. Disponível em: https://www.efdeportes.com/efd94/ deporte.htm. Acesso em: 14 Jun. 2020.

RAMÍREZ MACÍAS, Gonzalo. Estereotipos corporales en las portadas de los videojuegos de género deportivo. Revista Internacional de Medicina y Ciencias de la Actividad Física y el Deporte, v. 11, n. 42, p. 407-420, 2011. Disponível em: http://cdeporte.rediris.es/revistal revista42/artcuerpo213.htm. Acesso em: 14 jun. 2020.

SÁNCHEZ, Antonio; MARTÍNEZ CASTRO, Sonia Maria. Deporte y realidad virtual: la utopía lúdica humanizadora. In: SANCHO, Miguel Pablo; VIÑAO, Silvia (coord.). Impulso Humano. Murcia: Universidad Católica San Antonio, 2014. p. 16-58.

SÁNCHEZ , Antonio; DAVIS REMILLLARD, Joshua. eSport: Towards a Hermeneutic of Virtual Sport. Cultura, Ciencia y Deporte, v. 13, n. 38, 2018. DOI: https://doi.org/10.12800/ ccd.v13i38.1076 
SEGAL, David. Behind League of Legends, E-Sports's main attraction. New York Times, 10 oct, 2014. Disponível em: https://www.nytimes.com/2014/10/12/technology/riot-gamesleague-of-legends-main-attraction-esports.html. Accesso em: 14 Jun. 2020.

STAIANO, Amanda; CALVERT, Sandra. Exergames for physical education courses: Physical, social, and cognitive benefits. Child Development Perspectives, v. 5, n. 2, p. 9398, 2011. DOI: https://doi.org/10.1111/j.1750-8606.2011.00162.x

SUITS, Bernard. The elements of sport. In: MORGAN, William J. (ed.). Ethics in Sport. Champaign: Human Kinetics, 2007. p. 9 - 19.

TAMBURRINI, Claudio. Essays in the philosophies of sports. Goteborg: Acta Universitatis Gothoburgensis, 2000.

TAYLOR, T. L. Raising the Stakes: E-sports and the Professionalization of Computer Gaming. Cambridge, MA: MIT Press, 2012.

TAYLOR, T. L.; WITKOWSKI, Emma. This is how we play it: What a mega- LAN can teach us about games. FDG '10: In: Proceedings of the Fifth International Conference on the Foundations of Digital Games, 2010. p. 195-202. DOI: https://doi. org/10.1145/1822348.1822374

TORIL, Pilar; REALES, José M.; BALLESTEROS, Soledad. Video game training enhances cognition of older adults: A meta-analytic study. Psychology and Aging, v.29, n. 3, p. 706-716, 2014. DOI: https://doi.org/10.1037/a0037507

WAGNER, Michael. On the Scientific Relevance of eSports. In: Proceedings of the 2006 International Conference on Internet Computing \& Conference on Computer Games Development, ICOMP. Nevada: CSREA Press, 2006. p. 437-442.

WITKOWSKI, Emma. On the Digital Playing Field How We "Do Sport" With Networked Computer Games. Games and Culture, v. 7, n. 5, p. 349-374, 2012. DOI: https://doi. org/10.1177/1555412012454222 